\section{Apoyo social percibido en pacientes con Hipertensión Arterial y Diabetes Mellitus tipo II en Atención Primaria y su relación con autopercepción de salud}

\author{
FERNANDO POBLETE ${ }^{1}$, NICOLÁS BARTICEVIC ${ }^{2}$, \\ JAIME C. SAPAG ${ }^{1}$, PABLO TAPIA ${ }^{3, a}$, GABRIEL BASTÍAS ${ }^{1}$, \\ DIEGO QUEVEDO ${ }^{4, b}$, CAMILA VALDÉS $^{3 \mathrm{C}}$, CLAUDIA BUSTAMANTE $^{3, \mathrm{~d}}$, \\ CLAUDIA ALCAYAGA ${ }^{3, f}$, GABRIEL PÉREZ ${ }^{5}$
}

\section{Social support, self-rated health, treatment adherence and effectiveness in patients with type II diabetes and hypertension}

Background: A high level of social support (SS) is associated with better health outcomes in many conditions, such as chronic diseases. Aim: To describe the level of SS in patients with Hypertension and type II Diabetes at Primary Health Care level in Chile and its association with self-rated health, adherence to treatment and better glycemic and blood pressure control. Material and Methods: SS was measured using a social support inventory previously validated in Chile. Self-Rated Health was assessed with a single non-comparative general question; adherence to medication was assessed using the four-item Morisky medication adherence scale. Blood glucose and blood pressure control were also assessed. A logistic regression was performed to estimate Prevalence Odds Ratio (POR) and Robust Poisson method to estimate the Prevalence Ratio (PR). Results: Eighty three percent of the 647 participants evaluated high for SS. There was a significant correlation between SS and Self-rated health (POR 2.32; 95\% confidence intervals (CI) 1.19-11.23; PR 1.18; 95\% CI 1.07-1.31). No statistically significant association was observed with medication adherence, glycemic or blood pressure control. Conclusions: High levels of SS were found. The association between self-rated health suggests that SS interventions targeting vulnerable subgroups would be worthwhile.

(Rev Med Chile 2018; 146: 1135-1142)

Key words: Diabetes Mellitus, Type 2; Hypertension; Medication Adherence; Primary Health Care; Social Support.
'Departamento de Salud Pública, Escuela de Medicina Pontificia Universidad Católica de Chile. Santiago, Chile.

2Departamento de Medicina Familiar, Escuela de Medicina Pontificia Universidad Católica de Chile. Santiago, Chile.

${ }^{3}$ Escuela de Enfermería, Pontificia Universidad Católica de Chile.

Santiago, Chile.

${ }^{4}$ Centro de Salud Familiar Trinidad, Corporación Municipal de La Florida. Santiago, Chile.

${ }^{5}$ Centro de Salud Familiar La

Faena, Corporación Municipal de Peñalolén. Santiago, Chile.

assicólogo.

${ }^{b}$ Enfermero.

'Enfermera, Magíster en

Enfermería (c).

dEnfermera Matrona, Magíster en Enfermería.

eEnfermera Matrona, Magíster en Psicología.

Fuente apoyo financiero. Este estudio recibió el apoyo de la Comisión Nacional de Investigación Científica y Tecnológica (CONICYT) del Gobierno de Chile, a través del Programa FONDEF, con el financiamiento del proyecto ID ID15I10277 "Desarrollo de un modelo de atención para personas con Hipertensión Arterial y Diabetes tipo II basado en gestión del cuidado según ajuste de riesgo". El ente financiador no tuvo influencia en la recolección, procesamiento ni análisis de los datos, como tampoco en la elaboración del escrito.

Recibido el 25 de mayo de 2018, aceptado el 30 de agosto de 2018.

Correspondencia a: Nicolás Barticevic Lantadilla División de Salud Pública y Medicina Familiar Escuela de Medicina Pontificia Universidad Católica de Chile. Diagonal Paraguay 362 , segundo piso. Santiago, Chile. nicolas.barticevic@gmail.com 
$\mathrm{E}$ 1 apoyo social corresponde a una de las funciones críticas de las relaciones sociales y se ha asociado a beneficios en salud en distintos niveles. Su constructo se compone de cuatro dimensiones ${ }^{1-3}$, un apoyo instrumental (que implica provisión de ayuda tangible y servicios), apoyo informativo (provisión de información, consejo o sugerencias útiles para resolver problemas), apoyo valorativo (entrega de información a otro que es útil para autoevaluación) y apoyo emocional, que implica expresión de empatía, amor, confianza y preocupación. Además, el apoyo social puede ser provisto por una institución o persona ${ }^{4}$, y cuando es percibido como tal por quien lo recibe tiene mayor relación favorable con efectos en salud. Es decir, no es suficiente con entregar apoyo a una persona, sino que este apoyo debe ser experimentado como valioso y necesario por quien es el destinatario para que se relacione a efectos positivos ${ }^{5-7}$.

Entre las condiciones de salud a las que se ha relacionado el apoyo social con efectos positivos, destacan aquellas denominadas como crónicas, como el consumo de alcohol y otras sustancias psicoactivas $^{8}$, los problemas de salud mental ${ }^{9,10}$, las enfermedades cardiovasculares ${ }^{11-15}$, entre muchas otras. Asimismo, se ha observado efectos positivos en personas adultas mayores con problemas de funcionalidad ${ }^{16-18} \mathrm{y}$ morbilidad en múltiples áreas ${ }^{19}$. Los mecanismos por los que actuaría el apoyo social se relacionarían con efectos moduladores en la vía relacionada al estrés psicológico y fisiológico, así como en la modificación de conductas negativas para la salud ${ }^{20}$.

Por otro lado, el problema de las enfermedades crónicas y su enfrentamiento en el sistema de salud se ha transformado en una prioridad en todo el mundo ${ }^{21-23}$. En Chile, según análisis secundarios de la Encuesta Nacional de Salud (ENS) 2009-2010, la prevalencia de personas con más de una enfermedad crónica llega hasta $74,6 \%{ }^{24}$. Así mismo, algunas de las más frecuentes son parte de programas de atención en el ámbito ambulatorio ${ }^{25-27}$, como por ejemplo Hipertensión Arterial, Diabetes Mellitus II y Trastornos de la Salud Mental, con $27,6 \%, 12,7 \%$ y $15,2 \%$ (de síntomas depresivos) de prevalencias respectivamente, según los resultados preliminares publicados hasta el momento de la ENS 2016-201728.

Considerando lo anterior, se debe buscar alternativas de intervención que incluya la incorpo- ración del abordaje de factores o determinantes sociales, que pueden ser un importante mediador en la salud de las personas, y que complementen lo que ya se realiza en el sistema de atención. Esto, si bien es útil en toda la población de un sistema de salud, tanto público como privado, es particularmente relevante en los niveles socioeconómicos más desventajados, ya que son los que demuestran una mayor labilidad en salud, lo que está descrito Chile y el mundo por diversos estudios $^{28-31}$.

En consecuencia, con el objeto de avanzar en la comprensión local de este fenómeno, el presente estudio analiza la relación entre el apoyo social percibido de de pacientes del Programa de Salud cardiovascular (PSCV) en relación a la autopercepción de salud, la adherencia al tratamiento y el control de la Presión Arterial (PA) y Hemoglobina Glicosilada (HbAlc).

\section{Materiales y Métodos}

\section{Diseño y participantes}

Estudio trasversal descriptivo y analítico, realizado en un Centro de Salud de atención primaria de la Municipalidad de Peñalolén en el contexto de un estudio mayor, un ensayo clínico aleatorio controlado. Se invitó a participar a través de una selección aleatoria desde los registros de pacientes bajo control del PSCV, en particular aquellos con diagnósticos de Hipertensión Arterial (HTA) o Diabetes Mellitus II (DM II) de hasta 85 años de edad, sin problemas de comunicación para la aplicación de los instrumentos. Todos los participantes que aceptaron participar firmaron un consentimiento informado, aprobados por los respectivos comités de ética de la Pontificia Universidad Católica de Chile y del Servicio de Salud Metropolitano Oriente. Todos los procedimientos fueron concordantes con las normas éticas de la Declaración de Helsinki.

\section{Tamaño de muestra}

El tamaño de la muestra para el estudio se estableció a partir de dos criterios. Una probabilidad de evento de 0,5, para un IC de $95 \%$ y un margen de error de 5\%, por una parte; y superar el número mínimo de participantes para un Odds Ratio (OR) de 2 en la variable autopercepción en salud, con un poder estadístico de $80 \%$, para evaluar los resultados comparativos con la ro- 
bustez necesaria, vistas las dificultades de ello en la literatura del área. Así, el tamaño de muestra mínimo fue de 384 casos.

\section{Instrumentos y procedimientos}

Se aplicó una encuesta breve sociodemográfica. Para la evaluación de Apoyo Social se aplicó la encuesta MOS validada en Chile por nuestro grupo ${ }^{32}$. La encuesta consta de 19 ítems: el primero mide el tamaño de la red social y los restantes miden 4 dimensiones del apoyo social. El alfa de Cronbach para la totalidad de la escala fue de 0,89 ; y para las sub-dimensiones fue de 0,89 para interacción social positiva; 0,73 para apoyo afectivo; 0,78 para apoyo instrumental; $y$ 0,76 para apoyo emocional.

Para la estimación de pacientes en rango terapéutico, se midieron los niveles de PA y HbAlc. La PA fue medida por un técnico paramédico entrenado con un esfigmomanómetro certificado (Monitor de presión elite profesional Omron 7320 con brazalete $\mathrm{xl}$ ). Los niveles de HbAlc fueron medidos en sangre venosa extraída del brazo del paciente por un técnico paramédico y analizado en un único laboratorio certificado (laboratorio central de la Pontificia Universidad Católica de Chile). Para la medición de adherencia a tratamiento se aplicó el Test de Morisky-Green ${ }^{33}$, que ha sido utilizada en Chile sin registro de validación local, y con versión validada en español en pacientes hipertensos ${ }^{34}$. Para la evaluación de autopercepción de salud, se utilizó la pregunta general de autopercepción de salud no comparativa, similar a la utilizada en la ENS 2016-201728, demostrando que incluso a través de un solo ítem presenta un buen desempeño con relación al riesgo de morbilidad $y$ mortalidad ${ }^{35,36}$.

\section{Análisis de datos}

Se realizó análisis descriptivos de las variables, con tablas de frecuencias para variables categóricasy reporte de número de casos, medias, desviaciones estándar y medianas para las variables continuas. Se evalúan asociaciones mediante análisis bivariados por medio de pruebas de medias ( $\mathrm{t}$ de Student), de medianas (Mann-Whitney), chi cuadrado o de Fisher. Para análisis multivariados se analizan modelos de regresión logística para estimar OR y regresión de Poisson con varianza robusta para estimación de razón de prevalencias (RP). Los análisis se realizan con el programa SPSS $^{\circledast} 22.0$ para Windows y STATA 12.0.

\section{Resultados}

Se evaluó un total de 647 pacientes con una media de edad de 63 años (D Estándar de 12), $63,2 \%$ de sexo femenino (409), con $52,7 \%$ de pacientes diabéticos (341) de los cuales 269 (41,6\%) son además hipertensos. Más de $70 \%$ pertenecen a FONASA A o B. La mayoría posee hasta educación básica $(50,2 \%)$ o educación media $(41,8 \%)$. En la Tabla 1 se puede apreciar las características generales de los participantes.

El apoyo social en toda la muestra revela un nivel relativamente alto, con una media de 73 puntos y una mediana de 78 (máximo 90). Los niveles más bajos se obtienen al responder a cada ítem de acceso a apoyo social como "nunca" o "pocas veces" (preguntas 2 a la 18), lo que se traduce en un puntaje hasta 36. Esta situación se presenta en cerca de $3 \%$ de los pacientes. Al operacionalizar bajo apoyo social como aquellas encuestas con el total de respuestas de acceso a apoyo en las categorías de "de vez en cuando", o con máximo cuatro de las 18 preguntas en la categoría de "Casi siempre"

\section{Tabla 1. Caracterización de la población en estudio según variables principales}

\begin{tabular}{|lc|}
\hline & Valor \\
Parámetros clínicos & Media (DE) \\
HbA1c & $7,68(2,02)$ \\
PAS* & $134(20)$ \\
PAD & $81(12)$ \\
Resultados en salud & $\mathbf{n}(\%)$ \\
Baja autopercepción de salud & $342(52,85)$ \\
Baja adherencia al tratamiento & $316(48,84)$ \\
HTA fuera de rango terapéutico & $271(41,89)$ \\
DMIl fuera de rango terapéutico** & $180(54,4)$ \\
Estado civil & \\
Soltera/o & $116(17,92)$ \\
Casada/o y/o convive & $347(53,63)$ \\
Separado/viudo & $176(27,20)$ \\
Sin dato & $12(1,85)$ \\
Redes sociales & \\
Sin amigos cercanos & $251(38,79)$ \\
Un familiar cercano o ninguno & $85(13,1)$ \\
\hline
\end{tabular}

HbA1c: Hemoglobina glicosilada. DE: Desviación Estándar. *Diferencia significativa en PAS con menor promedio en sexo femenino en prueba $t(p=0,001)$. ${ }^{*} \mathrm{HbA} 1 \mathrm{c}$ presentan diferencias estadísticamente significativa $p \leq 0,001$ y $p \leq 0,044$ en la prueba de Mann-Whitney. Menor rango promedio en mujeres. 
, el apoyo bajo llega hasta 17\% aproximadamente. No hay diferencias significativas en la distribución del nivel de apoyo social total al comparar por sexo. En la Tabla 2 se muestra la distribución de los distintos componentes del apoyo social en la muestra, donde desataca la mayor proporción de apoyo alto en la dimensión apoyo emocional.

El análisis de la relación entre niveles altos de apoyo social y autopercepción de salud demostró ser significativo en el análisis bivariado y multivariado (ajustado por edad, sexo, estado civil, previsión y diagnóstico de depresión referido por el paciente), mostrando mejor autopercepción a mayor apoyo social percibido. Para las variables de adherencia o control de PA y HbA1c, no demostró una diferencia significativa entre los grupos el análisis bivariado y multivariado, ajustado por edad, sexo, estado civil y previsión en los modelos de regresión logística, como lo muestra la Tabla 3. En los análisis con regresión de Poisson con varianza robusta para estimar la RP se obtuvo tendencias similares, que no variaron con los ajustes de las distintas variables. Se realizó el mismo análisis para cada una de las cuatro dimensiones de apoyo de la escala con control de PA y Hb1Ac y adherencia, el que tampoco mostró resultados significativos.

Adicionalmente, se realizó análisis relacionando la disponibilidad de la red (reporte de amigos y familiares cercanos) con lo que no se observó diferencias significativas en los resultados.

Tabla 2. Distribución del nivel de apoyo según dimensión de apoyo social y sexo

\begin{tabular}{|lcccc|}
\hline Dimensión de apoyo social & $\begin{array}{c}\text { Alto } \\
\mathbf{n}(\%)\end{array}$ & $\begin{array}{c}\text { Femenino } \\
\text { Bajo } \\
\text { n (\%) }\end{array}$ & $\begin{array}{c}\text { Alto } \\
\mathbf{n ~ ( \% )}\end{array}$ & $\begin{array}{c}\text { Masculino } \\
\text { B (\%) }\end{array}$ \\
Interacción social positiva & $303(74,4)$ & $104(25,6)$ & $170(73,0)$ & $63(27,0)$ \\
Apoyo afectivo & $379(93,1)$ & $28(6,9)$ & $221(94,8)$ & $12(5,2)$ \\
Apoyo instrumental* & $284(69,8)$ & $123(30,2)$ & $201(86,6)$ & $31(13,4)$ \\
Apoyo emocional informativo & $279(68,6)$ & $128(31,4)$ & $154(66,1)$ & $79(33,9)$ \\
\hline Total & $332(81,6)$ & $75(18,4)$ & $196(84,5)$ & $36(15,5)$ \\
\hline
\end{tabular}

*Valor p menor de 0,001.

Tabla 3. Distribución del nivel de apoyo social según autopercepción de salud, adherencia al tratamiento y control de presión arterial y hemoglobina glicosilada

\begin{tabular}{|c|c|c|c|c|c|}
\hline & & $\begin{array}{c}\text { Alto Apoyo } \\
\text { Social* } \\
\text { n (\%) }\end{array}$ & $\begin{array}{c}\text { Bajo Apoyo } \\
\text { Social } \\
\text { n (\%) }\end{array}$ & ORP (IC 95\%)** & $\operatorname{RP}(\text { IC } 95 \%)^{* * *}$ \\
\hline $\begin{array}{l}\text { Autopercepción de } \\
\text { Salud }\end{array}$ & $\begin{array}{l}\text { Buena o muy buena } \\
\text { Regular o mala }\end{array}$ & $\begin{array}{l}268(50,6) \\
262(49,4)\end{array}$ & $\begin{array}{l}34(30,6) \\
77(69,4)\end{array}$ & $\begin{array}{c}2,32(1,19-11,23) \\
p=0,014\end{array}$ & $\begin{array}{c}1,18(1,07-1,31) \\
p=0,001\end{array}$ \\
\hline $\begin{array}{l}\text { Presión Arterial menor } \\
\text { a } 140 / 90 \mathrm{mmHg}\end{array}$ & $\begin{array}{l}\text { Sí } \\
\text { No }\end{array}$ & $\begin{array}{l}310(58,5) \\
220(41,5)\end{array}$ & $\begin{array}{l}62(55,9) \\
49(44,1)\end{array}$ & $0,93(0,49-1,74)$ & $1,02(0,93-1,13)$ \\
\hline $\begin{array}{l}\text { Adherencia al } \\
\text { tratamiento }\end{array}$ & $\begin{array}{l}\text { Sí } \\
\text { No }\end{array}$ & $\begin{array}{l}249(49,0) \\
259(51,0)\end{array}$ & $\begin{array}{l}51(48,1) \\
55(51,9)\end{array}$ & $0,78(0,41-1,55)$ & $0,99(0,89-1,09)$ \\
\hline $\begin{array}{l}\mathrm{HbA} 1 \mathrm{c} \text { en rango } \\
\text { terapéutico }\end{array}$ & $\begin{array}{l}\text { Sí } \\
\text { No }\end{array}$ & $\begin{array}{l}121(44,5) \\
151(55,5)\end{array}$ & $\begin{array}{l}27(50,9) \\
26(49,1)\end{array}$ & $0,73(0,3-1,77)$ & $0,97(0,88-1,07)$ \\
\hline
\end{tabular}

ORP: Odds Ratio de prevalencia. RP: Razón de prevalencias. *El nivel de apoyo social alto considera a todas aquellas personas con respuestas "casi siempre" o "siempre" en al menos 14 de las 18 preguntas del cuestionario. Todas las demás personas corresponden al grupo de bajo apoyo social. **Ajustado por edad, sexo, estado civil, previsión y diagnóstico de depresión referido por los pacientes *** Obtenido con regresión de Poisson con varianza robusta. Valores crudos, que no sufren cambios relevantes con los ajustes analizados. 


\section{Discusión}

El primer hallazgo relevante de los resultados de este estudio es que el apoyo social percibido es alto en la población en estudio, sin grandes diferencias por sexo, y que este estaría en buena parte explicado por la familia, ya que cerca de un tercio de los participantes no reporta amigos cercanos. Una explicación plausible, y que se ha descrito en la literatura por Berkman y colaboradores ${ }^{37}$, es que este grupo de pacientes por sus patologías crónicas ya poseen un mayor nivel de atención por parte del sistema de salud y de su familia más cercana, como es el caso chileno, ya que son parte de un programa estructurado con un paquete de prestaciones definidas. Este resultado comparado con la ENS 2010 para población general ${ }^{38}$, muestran más altos niveles de apoyo en general, incluyendo en el análisis de las sub-escalas. Lo anterior plantea la interrogante, en base a lo observado, respecto de cuánto mayor efecto puede aportar el diseño e implementación de nuevas intervenciones con orientación de apoyo social en este grupo de pacientes.

En la misma línea, la falta de relación entre apoyo social alto y parámetros de control en HTA y DMII, así como en adherencia a tratamiento, muestra consistencia con lo que refiere la literatura en relación a control de $\mathrm{PA}$ y $\mathrm{HbAlc}$, no así para la adherencia, donde se han reportado resultados mixtos en uno $\mathrm{u}$ otro sentido, aun cuando los meta-análisis más recientes sugieren asociación con adherencia ${ }^{4,6}$, en particular con las dimensiones no estructurales del apoyo social. Una posible explicación para nuestros resultados en el caso de adherencia podría estar en el instrumento de medición utilizado. Si bien el test de Morisky-Green ${ }^{33}$ es ampliamente utilizado por su facilidad de aplicación y corta extensión, hay otros instrumentos auto-referidos que muestran un mejor desempeño, pero a la vez mayor complejidad en su aplicación ${ }^{38}$ en el contexto de este estudio.

Por otro lado, las mediciones de Presión Arterial y de $\mathrm{HbAlc}$ fueron altamente estandarizadas $\mathrm{y}$, por lo tanto, muy robustas. El tamaño de muestra y el diseño también pueden explicar los resultados. Finalmente, se podría plantear que el instrumento no captura en profundidad el constructo de apoyo social, quedando fuera algún aspecto que pueda ser relevante de medir en nuestro contexto particular, como de alguna manera se evidenció en nuestro estudio de validación previo ${ }^{32}$ en relación al apoyo instrumental, que es justamente el que muestra menor nivel en nuestra medición y en la ENS.

En contraposición a lo anterior, la fuerte relación entre alto apoyo social y mejor autopercepción de salud plantea un espacio interesante para pensar en el valor potencial de intervenciones genéricas para aumentar el apoyo social y así obtener mejores resultados en salud general, más allá del problema de salud específico. Esta relación ha sido también observada en otros estudios ${ }^{35,36}$. Como se ha mencionado, la relación entre mejor autopercepción y mejores resultados en morbilidad y mortalidad ha sido estudiada ampliamente en la literatura ${ }^{39}$. Si bien el diseño de este estudio no permite controlar por todas las variables de confusión posibles, en el análisis multivariado se incorporaron las más relevantes, en particular el diagnóstico de depresión referido por los pacientes, considerando que este tipo de pacientes se encuentran en control habitual en los centros de salud, el antecedente del diagnóstico puede ser un dato aceptable y preciso para el análisis.

Este estudio posee limitaciones propias del diseño. Se trata de un estudio trasversal analítico, por lo que su mejor desempeño se encuentra en la descripción de eventos, como el caso del nivel de apoyo social en la población estudiada y los niveles generales observados para cada parámetro, para lo cual el poder es muy adecuado. El desempeño del diseño es menor para los análisis relacionales o comparaciones de parámetros entre subgrupos, como por ejemplo comparaciones de medias o proporciones de resultados entre subgrupos de mayor o menor apoyo social. Como se ha mencionado, la validez de los estimadores dependerá de varias consideraciones, como la presencia de factores de confusión no abordados en la medición, en el tipo de instrumentos usados, o en el análisis; también, las magnitudes de las diferencias o intensidad de la relación en estudio, donde el tamaño de muestra permitirá con mayor precisión estimar adecuadamente la significancia estadística de lo observado. Para minimizar los efectos de la medición, la estimación de control de $\mathrm{HbAlc}$ en Diabetes fue realizado de manera muy robusta en un solo laboratorio de alta calidad, asimismo, la PA fue medida por equipos de última generación, certificados para su uso y con menor dependencia del operador. Además, las encuestas utilizadas son 
validadas en nuestro medio ${ }^{32}$ o de amplio uso, como es el caso de autopercepción de salud. Sólo la medición de adherencia podría poseer menor nivel de validez. En los análisis, por otro lado, se incorporó en los modelos de regresión el ajuste por variables conocidas que pueden afectar los estimadores, de modo que si bien es posible que no se midiera o incorporara alguna co-variable relevante, ello parece poco probable.

En conclusión, el apoyo social percibido en pacientes con HTA y DMII muestra un nivel alto en lo general y en sus sub-componentes, y estaría en buena parte provisto por la familia. Si bien los resultados de este estudio sugieren que no estaría asociado a mejores resultados en control de PA y HbAlc y adherencia, al menos parte de estas conclusiones deben ser interpretadas a la luz de las limitaciones posibles del diseño. Por otro lado, la fuerte asociación entre alto Apoyo Social y mejor autopercepción de salud plantea como razonable el análisis más detallado de las intervenciones que hoy se aplican en los pacientes bajo control en los centros de salud primarios, con el fin de incorporar la perspectiva de apoyo social en el manejo del grupo que posee un menor nivel de apoyo en la comunidad.

Agradecimientos: Este estudio recibió el apoyo de la Comisión Nacional de Investigación Científica y Tecnológica (CONICYT) del Gobierno de Chile, a través del Programa FONDEF, con el financiamiento del proyecto ID ID15I10277 "Desarrollo de un modelo de atención para personas con Hipertensión Arterial y Diabetes tipo II basado en gestión del cuidado según ajuste de riesgo". Los autores agradecen a la Dirección de Salud y la Dirección del Centro de Salud Familiar La Faena, de la Corporación Municipal de Peñalolén, y a todos los participantes quienes hicieron posible este estudio.

\section{Referencias}

1. Berkman L, Glass T, Brissette I, Seeman T. From social integration to health: Durkheim in the new millennium. Social Science \& Medicine 2000; 51(6): 843-57. Disponible en: https://www.ncbi.nlm.nih.gov/pubmed/10972429 [Consultado el 22 de mayo de 2018].

2. Berkman L, Glass T. Social integration, social networks, social support and health. In Berkman L, Kawachi I, edi- tors. Social Epidemiology. New York: Oxford University Press; 2000. p. 137-73.

3. Heaney C, Israel B. Social networks and social support. In Glanz K, Rimer B, Viswanath K, editors. Health Behavior and Health Education: Theory Research and Practice. San Francisco: Josey-Bass; 2008.

4. Scheurer D, Choudhry N, Swanton K, Matlin O, Shrank W. Association Between Different Types of Social Support and Medication Adherence. The American Journal of Managed Care 2012; 18 (12): 461-7. Disponible en: http://www.ajmc.com/journals/issue/2012/2012-12vol18-n12/association-between-different-types-of-social-support-and-medication-adherence [Consultado el 18 de mayo de 2018].

5. Magrin M, D'Addario M, Greco A, Miglioretti M, Sarini M, Scrignaro M, et al. Social Support and Adherence to Treatment in Hypertensive Patients: A Meta-Analysis. Annals of Behavioral Medicine 2015; 49 (3): 307-18. Disponible en: https://www.ncbi.nlm.nih.gov/pubmed/25341642 [Consultado el 19 de mayo de 2018].

6. Vassilev I, Rogers A, Kennedy A, Koetsenruijter J. The influence of social networks on self-management support: a metasynthesis. BMC Public Health 2014; 14 (719). Disponible en: https://www.ncbi.nlm.nih.gov/ pmc/articles/PMC4223639/ [Consultado el 19 de mayo de 2018].

7. Tracy E, Munson M, Peterson L, Floersch J. Social Support: A Mixed Blessing for Women in Substance Abuse Treatment. Journal of Social Work Practice in the Addictions 2010; 10 (3): 257-82. Disponible en: https://www.tandfonline.com/doi/abs/10.1080/153325 6X.2010.500970 [Consultado el 20 de mayo de 2018].

8. Birtel M, Wood L, Kempa N. Stigma and social support in substance abuse: Implications for mental health and well-being. Psychiatry Research 2017; 252: 1-8. Disponible en: https://www.psy-journal.com/article/S01651781(16)30704-1/fulltext [Consultado el 18 de mayo de 2018].

9. Tajvar M, Fletcher A, Grundy E, Arab M. Social support and health of older people in Middle Eastern countries: A systematic review. Australian Journal on Ageing 2012; 32 (2). Disponible en: https://www.ncbi.nlm.nih.gov/ pubmed/23773244 [Consultado el 19 de mayo de 2018].

10. Fasihi T, Mohammad M, Dehghan T. The correlation of social support with mental health: A meta-analysis. Electronic Physician 2017; 9 (9): 5212-22. Disponible en https://www.ncbi.nlm.nih.gov/pubmed/29038699 [Consultado el 19 de mayo de 2018].

11. Stopford R, Winkley K, Ismail K. Social support and glycemic control in type 2 diabetes: a systematic review of observational studies. Patient Education \& Counseling 
2013; 93 (3): 549-58. Disponible en: https://www.ncbi. nlm.nih.gov/pubmed/24021417 [Consultado el 18 de mayo de 2018].

12. Marín-Reyes F, Rodríguez-Morán M. Apoyo familiar en el apego al tratamiento de la hipertensión arterial esencial. Salud Pública México 2001; 43 (4): 336-9. Disponible en: https://www.scielosp.org/article/ssm/ content/raw/?resource_ssm_path=/media/assets/spm/ v43n4/5900.pdf [Consultado el 18 de mayo de 2018].

13. Song Y, Nam S, Park S, Shin I, Jeong Ku B. The impact of social support on self-care of patients with diabetes: what is the effect of diabetes type? Systematic review and meta-analysis. The Diabetes Educator 2017; 43 (4): 396-412. Disponible en: https://www.ncbi.nlm.nih.gov/ pubmed/28578632 [Consultado el 18 de mayo de 2018].

14. Al-Dwaikat T, Hall L. Systematic review and critical analysis of measures of social support used in studies of persons with type 2 diabetes. Journal of Nursing Measurement 2017; 25 (2): 74-107. Disponible en: https:// www.ncbi.nlm.nih.gov/pubmed/28789742 [Consultado el 19 de mayo de 2018].

15. Graven L, Grant J. The Impact of Social Support on Depressive Symptoms in Individuals With Heart Failure: Update and Review. The Journal of Cardiovascular Nursing 2013; 28 (5): 429-43. Disponible en: https:// www.ncbi.nlm.nih.gov/pubmed/22728774 [Consultado el 19 de mayo de 2018].

16. Okura T, Heisler M, Langa K. Association Between Cognitive Function and Social Support with Glycemic Control in Adults with Diabetes Mellitus. Journal of the American Geriatrics Society 2009; 57 (10): 181624. Disponible en https://onlinelibrary.wiley.com/doi/ abs/10.1111/j.1532-5415.2009.02431.x [Consultado el 18 de nayo de 2018].

17. Strom J, Egede L. The Impact of Social Support on Outcomes in Adult Patients with Type 2 Diabetes: A Systematic Review. Current Diabetes Reports 2012; 12 (6): 769-81. Disponible en: https://www.ncbi.nlm.nih. gov/pmc/articles/PMC3490012/ [Consultado el 18 de mayo de 2018].

18. Kelly M, Duff H, Kelly S, McHugh Power J, Brennan S, Lawlor B, et al. The impact of social activities, social networks, social support and social relationships on the cognitive functioning of healthy older adults: a systematic review. Systematics Reviews 2017; 6 (1): 259. Disponible en: https://www.ncbi.nlm.nih.gov/ pubmed/29258596 [Consultado el 19 de mayo de 2018].

19. Pereira T, Pires N, Pires L, da Silva T, de Oliveira Y. Low supply of social support as risk factor for mortality in the older adults. Archives of Gerontology and Geriatrics 2017; 73: 77-81. Disponible en: https://www.ncbi.nlm. nih.gov/pubmed/28783514 [Consultado el 22 de mayo de 2018].

20. Kawachi I, Berkman L. Social ties and mental health. Journal of Urban Health 2001; 78 (3): 458-67. Disponible en: https://link.springer.com/article/10.1093\%2Fjurban\%2F78.3.458 [Consultado el 19 de mayo de 2018].

21. Zheng Y, Ley S, Hu F. Global aetiology and epidemiology of type 2 diabetes mellitus and its complications. Nature Reviews Endocrinology 2017; 14: 88-98. Disponible en: https://www.nature.com/articles/nrendo.2017.151 [Consultado el 18 de mayo de 2018].

22. Organización Mundial de la Salud. Informe mundial sobre la diabetes. Ginebra, Suiza: OMS; 2016. Disponible en: http://apps.who.int/iris/bitstream/handle/10665/254 649/9789243565255-spa.pdf?sequence $=1$ [Consultado el 18 de mayo de 2018].

23. Allotey P, Reidpath D, Yasin S, Chan C, de-Graft A. Rethinking health-care systems: a focus on chronicity. The Lancet. 2011; 377 (9764): 450-1 Disponible en: https:// www.ncbi.nlm.nih.gov/pubmed/21074257 [Consultado el 23 de mayo de 2018].

24. Margozzini P, Pizarro A, de Arcas M, Anriquez S, Jara L, Passi A. Multimorbilidad crónica en adultos chilenos: análisis de la Encuesta Nacional de Salud, ENS 20092010. Medwave 2016; 16 (6). Disponible en: http://www. medwave.cl/link.cgi/Medwave/ResumenesCongreso/ SP2016/EnfCronEnvDisc/6634.act [Consultado el 23 de mayo de 2018].

25. MINSAL. Guía Práctica Clínica: Tratamiento Farmacológico de la Diabetes Mellitus tipo 2. 2016-2017 [Online] Disponible en: http://www.redcronicas.cl/ wrdprss_minsal/wp-content/uploads/2014/04/DIABETES-MELLITUS-TIPO-2-1.pdf. [Consultado el 18 de mayo de 2018].

26. MINSAL. Guía clínica: Hipertensión Arterial Primaria o Esencial en personas de 15 años y más. [Online]. 2009. Disponible en: http://www.redcronicas.cl/wrdprss_minsal/wp-content/uploads/2014/04/Guía-Clínica-Hipertensión-Arterial-2010.pdf. [Consultado el 18 de mayo de 2018].

27. MINSAL. Orientación Técnica: Programa de Salud Cardiovascular. [Online]. 2017. Disponible en: http:// www.redcronicas.cl/wrdprss_minsal/wp-content/ uploads/2017/08/OT-PROGRAMA-DE-SALUD-CARDIOVASCULAR_05.pdf. [Consultado el 18 de mayo de 2018].

28. MINSAL-UC. Encuesta Nacional de Salud 2016-2017. Informe Final. 2017. Disponible en: http://www.encuestas.uc.cl/ens/img/ENS\%202016-2017\%20Informe\%20 final\%20V4.2.pdf. [Consultado el 19 de mayo de 2018].

29. Walker R, Smalls B, Campbell J, Strom-Williams J, 
Egede L. Impact of social determinants of health on outcomes for type 2 diabetes: a systematic review. Endocrine 2014; 47 (1): 29-48. Disponible en: https://www. ncbi.nlm.nih.gov/pubmed/24532079 [Consultado el 18 de mayo de 2018].

30. Clark M, Utz S. Social determinants of type 2 diabetes and health in the United States. World Journal of Diabetes 2014; 5 (3): 296-304. Disponible en: https://www. ncbi.nlm.nih.gov/pubmed/24936251 [Consultado el 18 de mayo de 2018].

31. Mejía-Lancheros C, Estruch R, Martínez-González M, Salas-Salvado J, Corella D, Gómez-Gracia E, et al. Nivel socioeconómico y desigualdades de salud en la prevención cardiovascular de la población española de edad avanzada. Revista Española de Cardiología 2013; 66 (10): 803-11. Disponible en: https://www.sciencedirect. com/science/article/pii/S0300893213002911 [Consultado el 19 de mayo de 2018]

32. Poblete F, Glasinovic A, Sapag J, Barticevic N, Arenas A, Padilla O. Apoyo social y salud cardiovascular: adaptación de una escala de apoyo social en pacientes hipertensos y diabéticos en la atención primaria chilena. Atención Primaria 2015; 47 (8): 523-31. Disponible en: https://www.sciencedirect.com/science/article/pii/ S0212656714003990?via\%3Dihub [Consultado de 19 de mayo de 2018].

33. Morisky D, Green L, Levine D. Concurrent and predictive validity of a self-reported measure of medication adherence. Med Care 1986; 24: 67-74.

34. Val A, Amorós G, Martínez P, Fernández M, León M. Estudio descriptivo del cumplimiento del tratamiento farmacológico antihipertensivo y validación del test de Morisky y Green. Atención primaria 1992; 10: 767-70.
35. Eriksson I, Undén A, Elofsson S. Self-rated health. Comparisons between three different measures. Results from a population study. International Journal of Epidemiology. 2001; 30 (2): 326-33. Disponible en: https://academic.oup.com/ije/article/30/2/326/713791 [Consultado de 19 de mayo de 2018].

36. Moreno X, Huerta M, Albala C. Autopercepción de salud general y mortalidad en adultos mayores. Gacela Sanitaria. 2013; 28 (3): 246-52. Disponible en: http:// scielo.isciii.es/pdf/gs/v28n3/revision.pdf [Consultado el 19 de mayo de 2018].

37. Berkman L, Blumenthal J, Burg M, Carney R, Catellier $D$, Cowan M, et al. Effects of Treating Depression and Low Perceived Social Support on Clinical Events After Myocardial Infarction. The Enhancing Recovery in Coronary Heart Disease Patients (ENRICHD) Randomized Trial. JAMA 2003; 289 (23): 3106-16.

38 Ministerio de Salud de Chile. Encuesta Nacional de Salud ENS Chile 2009-2010. Informe 2011 Disponible en: http://web.minsal.cl/portal/url/item/bcb03d7bc28b64dfe040010165012d23.pdf [Consultado el 20 de mayo de 2018].

39. Lam W, Fresco P. Medication Adherence Measures: An Overview. BioMed Research International. 2015 Octubre. Disponible en: https://www.hindawi.com/journals/ bmri/2015/217047/cta/ [Consultado el 20 de mayo de 2018].

40. White A, Philogene G, Fine L, Sinha S. Social Support and Self-Reported Health Status of Older Adults in the United States. American Journal of Public Health 2009; 99 (10): 1872-8. Disponible en: https://www.ncbi.nlm. nih.gov/pmc/articles/PMC2741527/ [Consultado el 22 de mayo de 2018]. 